\title{
OPEN Capturing hidden regulation based on noise change of gene expression level from single cell RNA-seq in yeast
}

Thoma Itoh ${ }^{1} \&$ Takashi Makino ${ }^{1,2 \bowtie}$

Recent progress in high throughput single cell RNA-seq (scRNA-seq) has activated the development of data-driven inferring methods of gene regulatory networks. Most network estimations assume that perturbations produce downstream effects. However, the effects of gene perturbations are sometimes compensated by a gene with redundant functionality (functional compensation). In order to avoid functional compensation, previous studies constructed double gene deletions, but its vast nature of gene combinations was not suitable for comprehensive network estimation. We hypothesized that functional compensation may emerge as a noise change without mean change (noise-only change) due to varying physical properties and strong compensation effects. Here, we show compensated interactions, which are not detected by mean change, are captured by noise-only change quantified from scRNA-seq. We investigated whether noise-only change genes caused by a single deletion of STP1 and STP2, which have strong functional compensation, are enriched in redundantly regulated genes. As a result, noise-only change genes are enriched in their redundantly regulated genes. Furthermore, novel downstream genes detected from noise change are enriched in "transport", which is related to known downstream genes. Herein, we suggest the noise difference comparison has the potential to be applied as a new strategy for network estimation that capture even compensated interaction.

\author{
Abbreviations \\ GRN Gene regulatory network \\ TF Transcription factor
}

Cells are controlled and maintained by proteins translated from thousands of genes. Genes involved in cellular function interact with each other to exert their actions in cells ${ }^{1}$. To understand the regulatory mechanisms of gene expression, the regulatory relationships among genes have been closely examined ${ }^{2}$. Gene regulatory networks (GRNs) are directed graphs showing regulatory relationships of transcription factors (TFs) and their target genes. Since the GRN plays an important role in connecting genes and phenotypes, uncovering interactions contributes to understanding diverse biological phenomena. In recent years, a vast amount of expression data has rapidly accumulated through the development of high-throughput RNA-seq, resulting in data-driven GRN estimation methods, which have drawn much attention. Most GRN estimation methods are based on the assumption that perturbations in a specific gene affects downstream genes ${ }^{3}$. Capturing changes in expression levels due to the deletion of a specific gene effectively estimates molecules downstream of the deleted gene. However, the actual GRN is more complex, and may not be estimated with this simple assumption. Hundreds of genes downstream of STP2, which is a TF, have been validated by experimental approaches. Interestingly, most were detected from the double deletion of STP 1 and $S T P 2^{4,5}$, and did not show a change in the mean expression level when only STP2 was deleted ${ }^{6}$. This phenomenon would be explained by functional compensation of a STP2 paralog. Since the lost function, upon STP2 deletion, was compensated by $S T P 1$, which is the functionally redundant gene with STP2, the downstream genes of STP2 are not affected by the STP2 deletion ${ }^{7}$, resulting in no change in the expression levels of STP2 downstream genes.

\footnotetext{
${ }^{1}$ Department of Biology, Faculty of Science, Tohoku University, 6-3, Aramaki Aza Aoba, Aoba-ku, Sendai 980-8578, Japan. ${ }^{2}$ Graduate School of Life Sciences, Tohoku University, 6-3, Aramaki Aza Aoba, Aoba-ku, Sendai 980-8578, Japan. ${ }^{\circledR}$ email: tamakino@tohoku.ac.jp
} 


\section{WT}
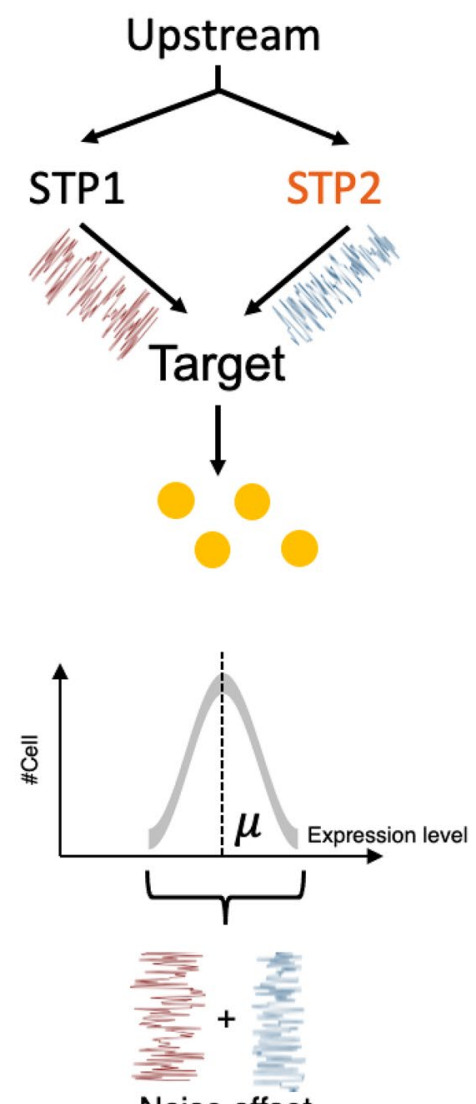

\section{$\triangle$ STP2}
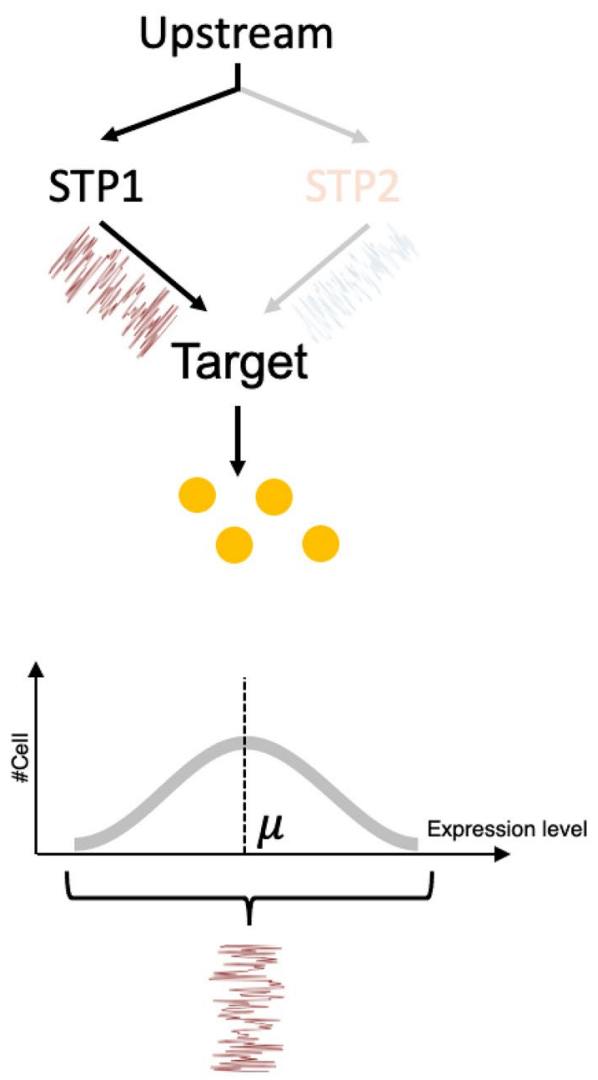

No noise offset

Figure 1. Noise difference detects functional compensation. STP1 and STP2 redundant pathway (Left: Wildtype, Right: STP2 deletion mutant). The lack of STP2 is compensated by STP1, resulting in no change in the mean expression level of downstream genes (orange circles). However, expression noise that propagated from upstream changes differ due to changes in physical characteristics of upstream genes. An example of change of distribution is shown at the bottom. The vertical axis represents the number of cells and the horizontal axis shows the expression level of the target gene. Whereas the variance was small because of the noise offset by the redundant pathway in the WT, the variance became large due to the lack of noise offset in $\triangle \mathrm{STP} 2$. Mean remains constant by the functional compensation effect. Note that whether the noise becomes large or small upon deletion is not evident, and that the direction of change is not an essential issue in this study.

While functional compensation provides robustness to perturbations in the GRN, it hinders estimation of downstream genes. To identify downstream genes that are not affected by upstream perturbations due to functional compensation, we focused on a change in expression noise by gene deletion. Expression noise is the stochastic variation in gene expression quantified by variance excluding the contribution of the mean in a clonal population $^{8}$. Since expression noise is affected by the transcription or translational efficiency characterized by the physical property of a sequence ${ }^{9}$, even functionally redundant gene pairs may show different expression noise. Furthermore, since it has been reported that expression noise propagates from upstream genes, the expression noise altered by compensation of redundant genes is expected to propagate to downstream genes and alter the expression noise of downstream genes ${ }^{9}$. Based on these previous reports, we hypothesize that noise change can be observed downstream of functionally compensated genes due to the physical change in upstream genes (Fig. 1).

Notably, not all noise changes imply functional compensation, as noise change reflects changes in physical properties, such as upstream translational efficiency, and does not necessarily indicate functional compensation. If functional compensation occurs upstream, the genes must show noise change without mean change (noiseonly change), due to changes in physical properties and compensation effects on the expression level. Therefore, we focused on genes that show noise-only change and investigated whether they are enriched in the common downstream genes of redundant gene pairs, such as STP1/2 (Fig. 2a).

In this study, we aimed to estimate mean-change genes and noise-change genes in yeast using scRNA-seq data by comparing expression patterns in a single deletion mutant $(\Delta \mathrm{STP} 1, \Delta \mathrm{STP} 2, \Delta \mathrm{RTG} 1, \Delta \mathrm{RTG} 3, \Delta \mathrm{GLN} 3$, $\triangle$ GAT1, $\triangle$ GZF3, and $\triangle$ DAL80; Table S1) with those in wildtype. Furthermore, we investigated enrichment of 
estimated genes to the downstream genes shared by a gene group, such as paralogous pair or protein complex members (Fig. 2a).

\section{Results and discussion}

Redundantly regulated target; STP1 and STP2. STP1 and STP2 are a duplicated pair of genes that arose from whole genome duplication (ohnologs), and thus share many downstream genes. Since it has been reported that STP1 and STP2 have redundant functions ${ }^{7}$, those common targets are likely to be redundantly regulated. Regarding the downstream genes shared by STP1/2 (STP1 and STP2), the proportion of genes that show mean expression change in the $\triangle \mathrm{STP} 1$ and $\triangle \mathrm{STP} 2$ was $36 \%(133 / 374)$ and $24 \%(91 / 374)$, respectively. The remaining genes did not show mean expression change in the single deletion, which suggests functional compensation $^{4-6}$ (Fig. 2b). Based on our hypothesis, those redundantly regulated genes can be detected by noiseonly change, even in the single deletion of STP1 or STP2.

First, we estimated mean-change genes and noise-change genes using the BASiCS R package. Mean and noise were inferred by comparing the gene expression of WT with that of the interest strain based on the hierarchical Bayesian model proposed in previous studies ${ }^{10,11}$. The relationship between inferred mean change and noise change is shown in Fig. 2c-1, and the transition of the inferred mean and noise is shown in Fig. 2c-2. From those relationships, we extracted noise-only change genes (Fig. 2c-1, blue dots) and mean-change genes (Fig. 2c-1, red dots).

We examined whether mean-change genes or noise-only change genes in $\triangle \mathrm{STP} 1$ and $\triangle \mathrm{STP} 2$ were enriched in the redundant downstream genes. Note that we consider the downstream genes shared by the functionally redundant genes (e.g., STP1 and STP2) as the redundant downstream genes. The mean change genes upon $\triangle \mathrm{STP} 2$ were not enriched in the downstream genes shared by $S T P 1 / 2$ (Fig. 3a). This result was consistent with a previous study showing that most downstream genes of STP2 were undetectable from single deletion of $S T P 2^{6}$. However, mean change genes upon $\triangle \mathrm{STP} 1$ were enriched in the downstream genes shared by $S T P 1 / 2(p<0.05$, Fisher's exact test; Fig. $3 \mathrm{a}$ ). This result suggests that the deletion of STP1 was not well compensated by STP2, although a previous study reported that deletion of $S T P 1$ was compensated by $S T P 2^{7}$. Subsequently, we quantified noise considering heterogeneity due to cell cycle. Briefly, we clustered WT and mutant cells simultaneously by similar expression pattern and executed comparison within each clusters (Fig. S1, S2 and see Material and methods). Because this analysis is vulnerable to the clustering methods, we keep this analysis as supplement. When we executed the same analysis with Fig. 3a but with considering cell cycle heterogeneity (Fig. S4a and see Materials and Methods), the same tendency with Fig. 3a were maintained. On the contrary, the noise-only change genes were enriched in the downstream genes shared by $S T P 1 / 2$ in both $\Delta$ STP1 and $\Delta$ STP2 $(p<0.05$, Fisher's exact test; Fig. 4a). This result supports our hypothesis that the downstream genes of functionally redundant genes show noise-only change. When we eliminated cell cycle heterogeneity, the enrichment of noise-only change genes to common downstream genes of $S T P 1 / 2$ disappeared in $\triangle S T P 1$, but a relatively high proportion of noise-only change genes (2/12) were common downstream genes of STP1/2 (Fig. S5a). Note that in the above results, we treated with the downstream genes shared by the $S T P 1 / 2$ as the redundant targets and executed an enrichment test toward those genes. From a more conservative perspective, it is possible to consider the redundant targets as the downstream genes detected only from the experiment using the double deletion, $\triangle$ STP $1 \Delta$ STP2. We executed the analysis based on this consideration regarding redundant targets. The results are shown in the Fig. S3. The same tendency as those obtained in the main results were observed for $\Delta \mathrm{STP} 2$, but the significant enrichment of the noise-only change for $\triangle$ STP1 was not observed. Since in this analysis many of the true redundant genes appeared to be missing among the conservatively defined redundant genes, the consistent results regarding $\triangle \mathrm{STP} 2$ suggests that these findings are highly reliable.

Non redundantly regulated target; RTG1 and RTG3. Next, as control, we investigated downstream genes shared by non-redundant genes. $R T G 1$ and $R T G 3$ are not a paralog pair, but they have significant overlap in downstream genes to form a complex ${ }^{6}$. We investigated whether mean change genes or noise-only change genes were enriched in the downstream genes shared by $R T G 1 / 3$. The mean change genes were enriched in the common downstream genes in both $\triangle$ RTG1 and $\triangle$ RTG3 $(p<0.05$, Fisher's exact test; Fig. $3 b)$. The results are reasonable because $R T G 1$ and $R T G 3$ do not have functional compensation and the perturbation of even one gene produces downstream effects. We observed the same trend even when we considered cell cycle heterogeneity (Fig. S4b). The noise-only change genes were not enriched in $\triangle$ RTG1 nor $\Delta$ RTG3 (Fig. 4b). This is also consistent with the result considering cell cycle heterogeneity (Fig. S5b). These results suggest that RTG1 and RTG3 do not exhibit functional compensation.

Possibly redundantly regulated target; GATA family. Next, we focused on the GATA family, GAT1, GZF3, DAL80, and $G L N 3^{12}$, and investigated networks constituted from homologous gene groups. They are paralogs and possibly have redundant functions. We investigated whether GATA family members display functional compensation by estimating whether noise-only change genes are enriched in common downstream genes of the paralogous family. In $\triangle \mathrm{GZF} 3$, the mean change genes were enriched in downstream genes shared by GZF3 and other GATA family members $(p<0.05$, Fisher's exact test; Fig. 3c). However, other deletion strains from the GATA family did not show enrichment of mean change genes compared to common downstream genes. When we considered cell cycle heterogeneity, any genes did not show the enrichment (Fig. S4c). The noise-only change genes were enriched in the common downstream genes of the GATA family only in $\triangle$ GLN3 $(p<0.05$, Fisher's exact test; Fig. 4c). Although a redundant pathway has not been reported between GLN3 and other genes, double deletion of GLN3 and GAT1 or GLN3 and DAL80 decreased growth rate ${ }^{13,14}$. These results suggest the existence of functional compensation between GLN3 and GAT1 and/or GLN3 and DAL80. In Fig. 
(a)

1. scRNA-seq data from deletion strains

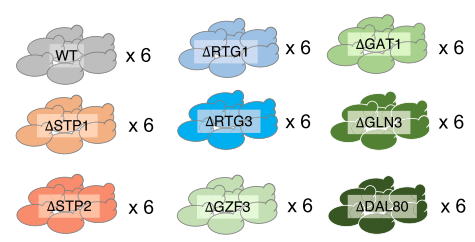

2. Select two deletion strains that share downstram genes

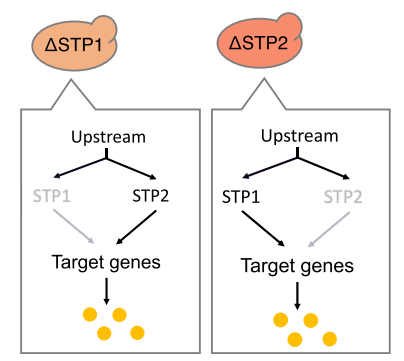

3. Filtering technical noise

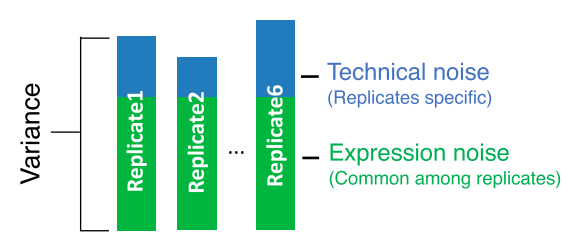

4. Difference test in mean and noise

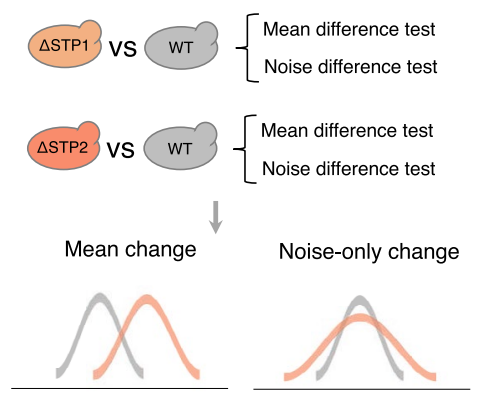

5. Enrichment anaysis
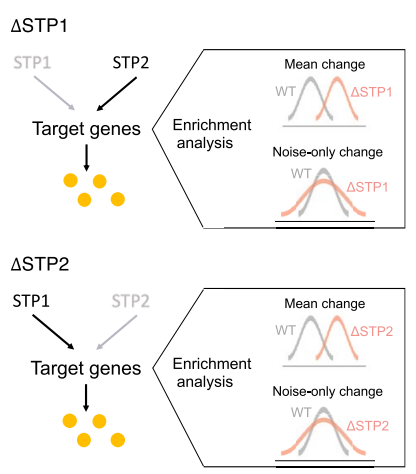

(b)

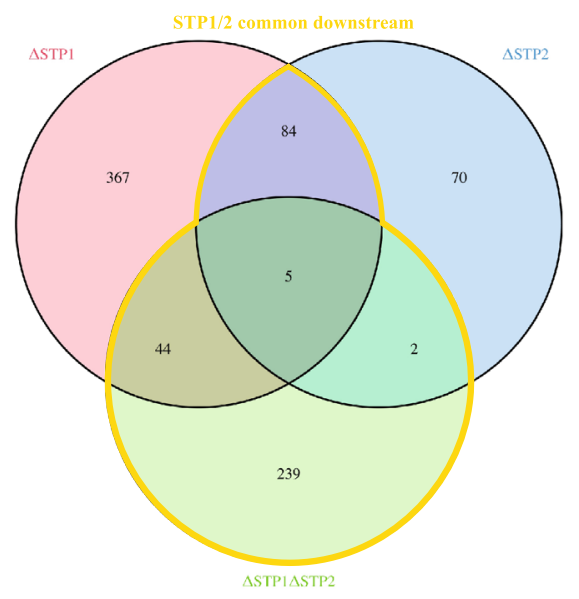

(c)

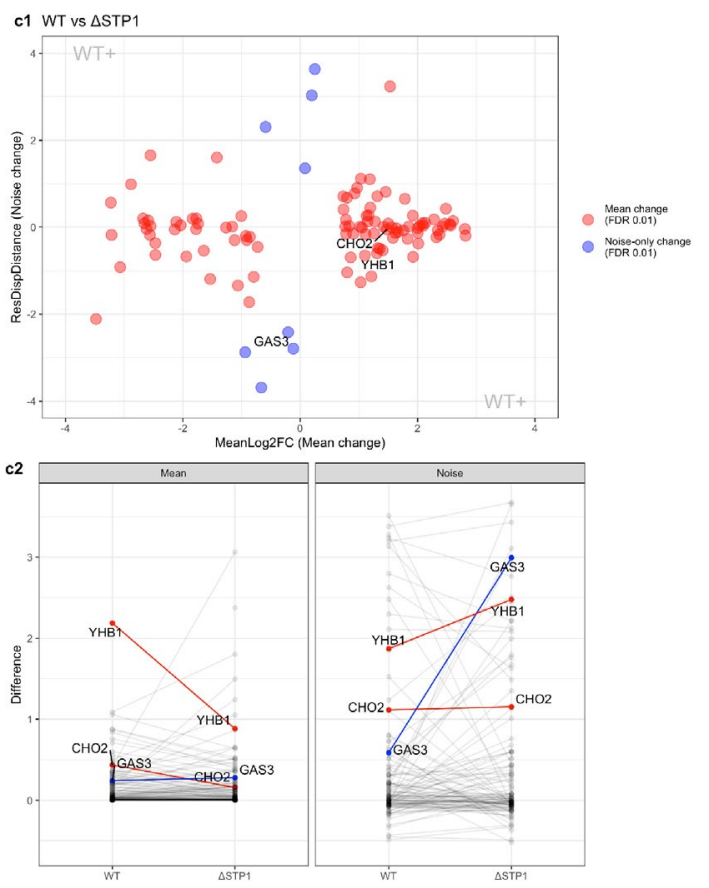


4Figure 2. Schematic workflow and data processing. (a) Schematic workflow. 1) scRNA-seq data from wildtype and deletion strains. Each strain has 6 replicates that were separately constructed. 2) We selected two deletion strains that share a common downstream gene. Although STP $1 / 2$ are depicted, RTG1/2 and GATA family (GZF3, GAT1, GLN3, and DAL80) were selected as pair (group) for the same reason. 3) Technical noise is eliminated by decomposing the total variance to technical noise and expression noise using the hierarchical Bayesian model. Briefly, common variance among replicates is recognized as expression noise, and replicate specific variance is recognized as technical noise. 4) Comparison between wildtype and the interest strain regarding mean and noise. Further, we derived the noise-only change genes that show significant noise change without mean change. An example of distribution change is shown at the bottom. 5) Investigation of the enrichment of mean change or noise-only change genes into the downstream genes shared by the pair genes (STP1/2, in this example). (b) Venn diagram of STP1/2 downstream genes. The pink and blue areas represent genes that showed mean change in the single deletion, $\Delta$ STP1 (500) and $\Delta$ STP2 (161) respectively. The green area represents genes that showed mean change in the double deletion $\Delta$ STP1 $\Delta$ STP2 (290). The area circled in yellow indicates the downstream genes shared by STP1/2 (374). (c) Comparison of mean and noise change genes in WT vs $\triangle \mathrm{STP} 1$. c1) $\mathrm{x}$-axis: mean expression change measured by log2 fold change (Mean Log2FC). $y$-axis: expression noise change measured by the distance of residual over-dispersion (ResDispDistance), which is the noise difference correcting the mean dependency. The red dots represent genes with at least mean change (e.g., CHO2, YHB1). The blue points represent genes with noise-only change which is the noise change without mean change (e.g., GAS3). c2) Left: mean expression level transition. Right: expression noise transition. YHB1 and $\mathrm{CHO} 2$ are the mean change genes and are showed by the red line. YHB1 shows both mean change and noise change. $\mathrm{CHO} 2$ shows only mean change without noise change. GAS3 is the noise-only change gene and is showed by the blue line. Whereas GAS3 showed significant noise change, mean expression was not changed. Schematic workflow for enrichment analysis of mean change genes and noise-only change genes to the target.

S5c, any strains did not show enrichment with common downstream genes with the GATA family even when we considered cell cycle heterogeneity. The small number of common downstream genes might hinder the estimation of downstream genes.

In summary, we found that downstream genes shared by a functional compensative pair tend to show noiseonly changes when one of the functional compensative pairs, such as STP1 and STP2, is deleted. Although other functional compensative pairs should be used to reinforce our results, no suitable scRNA-seq data are available to perform additional examinations. scRNA-seq of yeast is still an advancing technology and the data we require is expected to accumulate from now on.

From the above results, we suggest that noise-only change captures redundantly regulated genes. Based on our concepts, the candidate of redundantly regulated genes, which was detected by noise-only change, must be common to the deletion strain of the redundant gene pair. In fact, we detected three genes (MUP1, CDC21, GAS3) that show noise-only change in both $\triangle$ STP1 and $\triangle$ STP2 (genes indicated by two dark red or blue arrows in Fig. 5), while two of these (MUP1, CDC21) are known as shared downstream genes of STP1 and STP2 (genes indicated by two dark red arrows in Fig. 5). We propose that GAS3 is a new redundantly regulated candidate of $S T P 1 / 2$. Although an interaction between STP2 and GAS3 has not been reported, an interaction between STP1 and GAS3 was reported ${ }^{6}$. This supports that GAS3 is a new redundantly regulated candidate by $S T P 1 / 2$. Notably, although the number of noise-only change genes common to $\triangle \mathrm{STP} 1$ and $\triangle \mathrm{STP} 2$ was three, the number of mean change genes common to $\triangle \mathrm{STP} 1$ and $\triangle \mathrm{STP} 2$ was zero. These results support our hypothesis that redundantly regulated genes that cannot be detected from mean change but are detected from noise-only change.

However, not all noise-only change genes are common to $\triangle \mathrm{STP} 1$ and $\triangle \mathrm{STP} 2$. The total number of noise-only change genes in $\triangle \mathrm{STP} 1$ is 15 , while there are eight in $\triangle \mathrm{STP} 2$. As described above, the three genes overlap. Most noise-only change genes are detected only in $\triangle \mathrm{STP} 1$ or $\triangle \mathrm{STP} 2$. We speculate that this asymmetric noise-only change is caused by (1) artifact of FDR threshold, (2) asymmetric compensation, or (3) compensation by other genes. First, since the extent of noise difference caused by gene deletion is attributed to physical properties of the deleted gene, such as translational efficiency ${ }^{9}$, the threshold must differ gene by gene. Applying the same cut off to all genes leads to increased false negative results. The precise modeling of noise propagation must improve detection of redundant regulation. Second, asymmetric functional compensation might be considered. For example, the deletion of STP2 is compensated by STP1, but the deletion of STP1 is not compensated by STP2 with fewer interactions. In such situations, the gene shows mean change upon $\triangle$ STP1 and on the contrary the gene shows noise-only change upon $\triangle \mathrm{STP} 2$. Third, functional compensation by other genes might be considered. We assumed that functional compensation is likely to occur between paralogous pairs due to their functional similarity, but there are many synthetic effects between STP $1 / 2$ and other genes ${ }^{13}$. However, a synthetic effect does not necessarily translate to functional compensation, but those interactions suggest that a lack of STP1 or STP2 functionality may possibly be compensated by the other genes.

Novel downstream candidates of STP1 and STP2 detected from noise change. We investigated noise change genes, including mean change, to determine whether noise difference tests narrow down the reasonable downstream genes. We hypothesized that noise-only change genes shared by the deletion strain of a paralogous pair, such as $\triangle \mathrm{STP} 1$ and $\triangle \mathrm{STP} 2$, are likely to be the target of functional compensation. However, the noise change genes accompanied with mean change genes also can be recognized as downstream gene candidates. Interestingly, both known STP2 downstream genes and novel STP2 downstream candidates detected from noise change were enriched in transport-related genes ( $p<0.01$; SGD GO Term Finder; Process; Table 1). Therefore, it can be argued that the noise difference test has targeted reasonable candidates. 
a

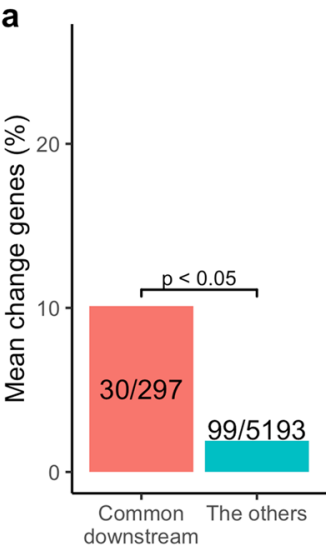

STP1

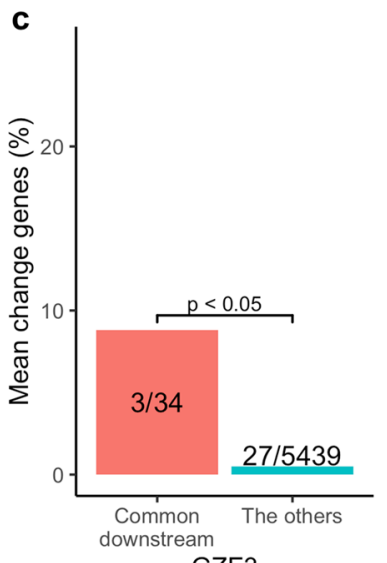

GZF3

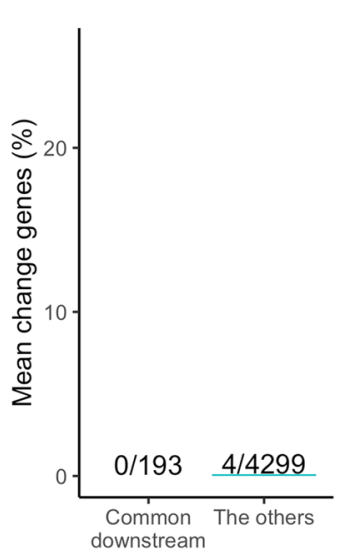

STP2

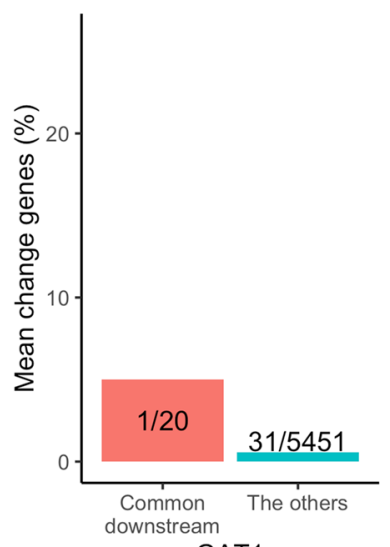

GAT1

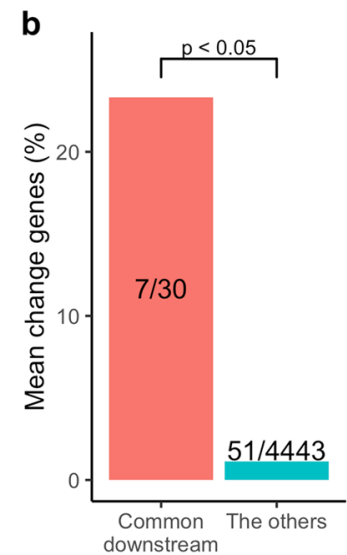

RTG1
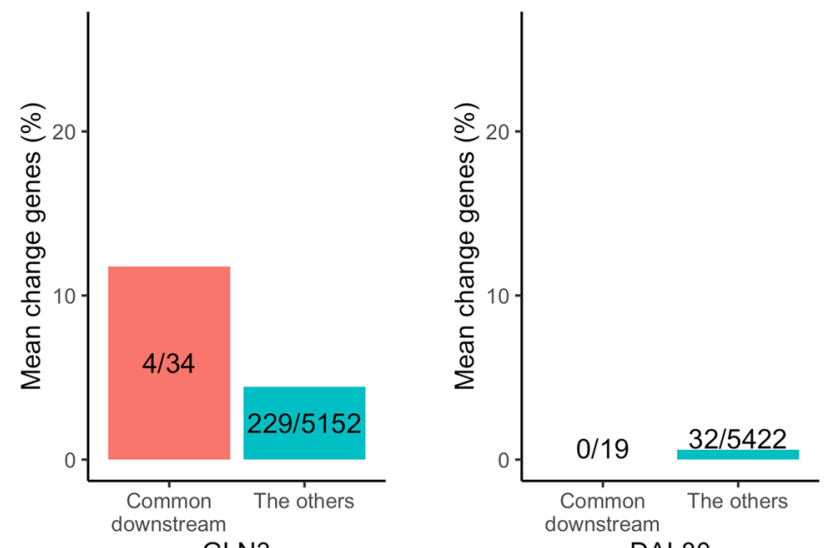

GLN3

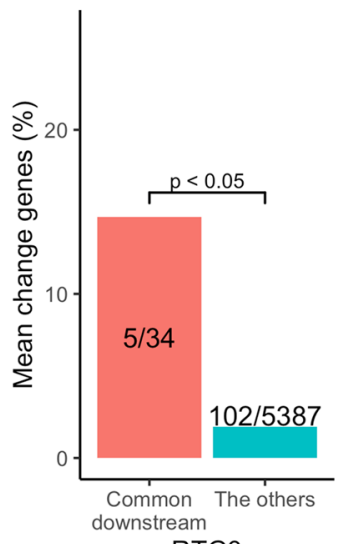

RTG3

DAL80

Figure 3. Ratio of mean change genes in known downstream genes shared by homologous groups. Bars show the proportion of mean change genes in the two categories labeled in the $\mathrm{x}$-axis. Common downstream; the reported downstream genes that are shared by the gene groups in which the deleted gene belongs. The others; the other genes in the right category. Bars indicate the ratio of mean change genes in each category. Note that the numbers of common downstream genes (shown on the red bar) do not coincide (even within the group) because non expressed genes in each strain are excluded. (a) Redundant gene group; STP1 and STP2. (b) Non redundant gene group; RTG1 and RTG3. (c) Possibly redundant paralogous group; GATA family (DAL80, GAT1, GLN3, and GZF3). The $p$-values above each bar were calculated using Fisher's exact test.

In this study, we executed two types of analysis. (1) The conservative difference test, which quantified values from all cells (Figs. 3 and 4), and (2) the progressive difference test, which quantified values from cells belonging to the same cluster (Fig. S4 and S5). In the later progressive analysis, as cell cycle heterogeneity is excluded from noise quantification, we can quantify that the noise change originated from a molecular process (but the results are affected by clustering). In the former conservative analysis, CLN1, which is related to cell cycle regulation and does not interact with $S T P 1$, showed noise change upon $\triangle \mathrm{STP} 1$, but in the later progressive analysis, CLN1 did not show noise change upon $\triangle \mathrm{STP} 1$ (Table S2). In the same way, CLN2, which is related to the cell cycle and does not interact with STP2, showed noise change upon $\triangle \mathrm{STP} 2$, but when we consider cell cycle heterogeneity, CLN2 did not show significant noise change (Table S2). This implies that the noise change of CLN1 and CLN2 originated from differences in cell cycle heterogeneity that are out of our interests. We consider that the consistent results of conservative and progressive analysis should be reliable. We found four consistent genes in $\triangle \mathrm{STP} 2$ that show noise change, both from conservative and progressive analysis (CDC21, SIT1, FIT2, FIT3). FIT2 and CDC21 are known as shared downstream genes of STP1 and STP2 (genes indicated by two dark red arrows in Fig. 5). SIT1 and FIT3 are possibly newly downstream candidates of STP2, and function as transporters, similar to the many known downstream genes of STP2. In addition, SIT1 and FIT3 are known as downstream genes of STP1 (genes indicated by dark red arrows and blue arrows in Fig. 5). This suggests they are redundantly regulated by STP1 and STP2. Note that SIT1 and FIT3 are the noise-only change genes.

In summary, we detected four novel gene candidates that are redundantly regulated by STP1 and STP2 (ARG1, FIT3, SIT1, GAS3), even from the single deletion strain of STP2 (Genes indicated by red arrow and blue arrow in Fig. 5). Three of these (FIT3, SIT1, GAS3) are constant, as we observed consistent results across progressive and conservative analyses. Interestingly, $A R G 1$ is involved in the arginine biosynthesis process ${ }^{15}$, and it was 


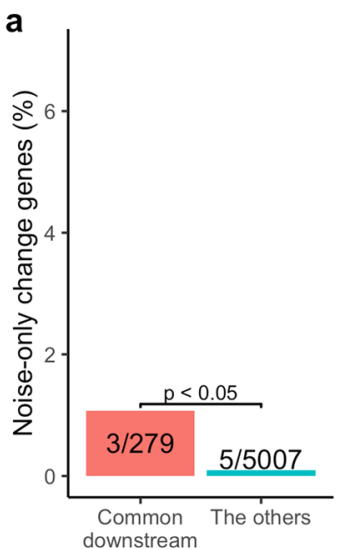

STP1

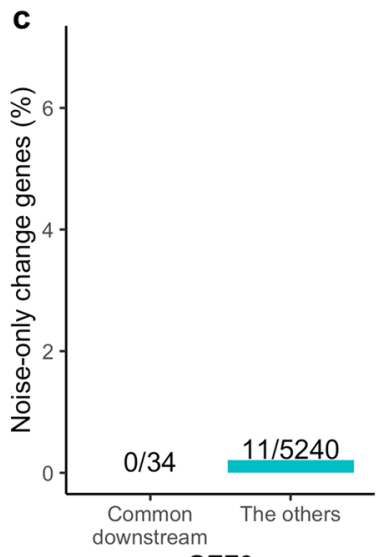

GZF3

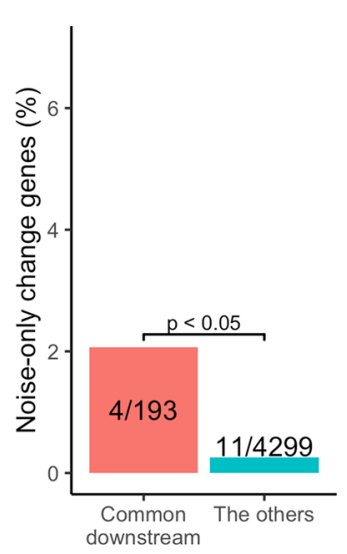

STP2

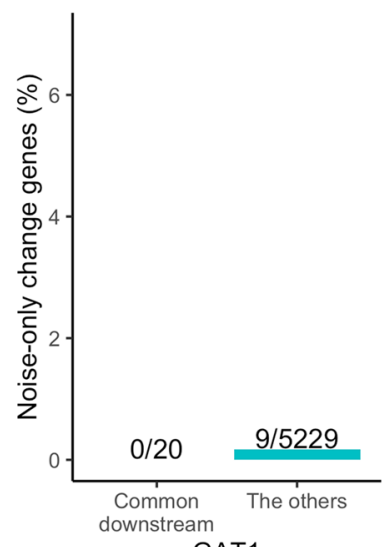

GAT1
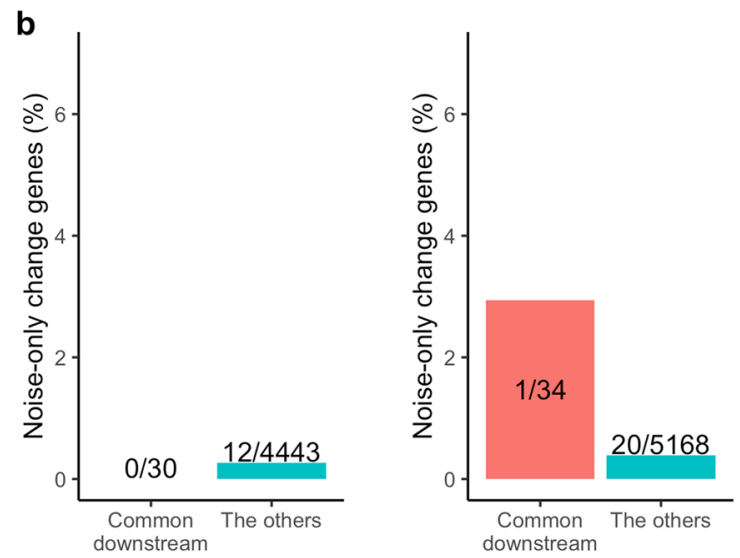

RTG1
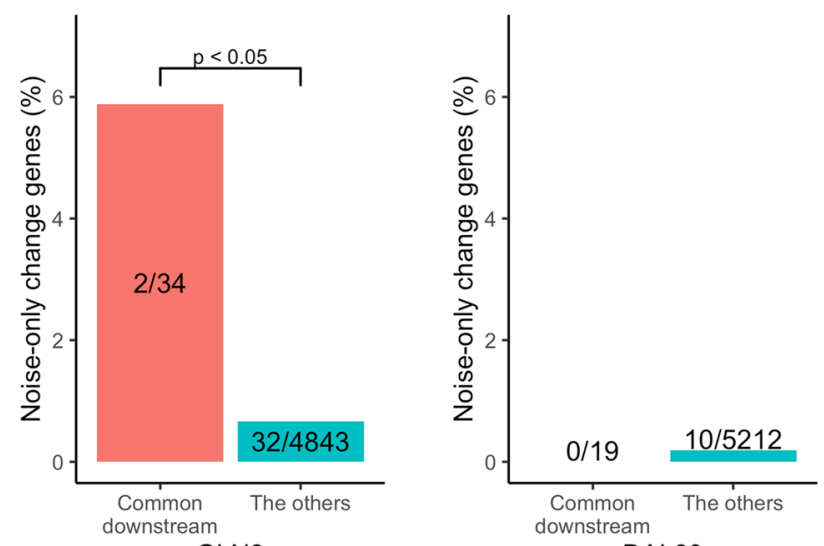

GLN3

DAL80

Figure 4. Ratio of noise-only change genes in known downstream genes shared by homologous groups. Bars show the proportion of noise-only change genes in the two categories labeled in the $\mathrm{x}$-axis. Common downstream; the reported downstream genes that are shared by the gene groups in which the deleted gene belongs. The others; the other genes in the right category. Bars show the ratio of noise-only change genes in each category. Note that the numbers of common downstream genes (shown on the red bar) do not coincide (even within group) because non expressed genes in each strain are excluded. (a) Redundant gene group; STP1 and $S T P 2$. (b) Non redundant gene group; RTG1 and RTG3. (c) Possibly redundant paralogous group; GATA family (DAL80, GAT1, GLN3, and GZF3). The $p$-values above each bar were calculated using Fisher's exact test.

previously unknown that STP2 affects this process. Thus, our results suggest that STP2 may be involved in the arginine biosynthesis process.

Based on the above results, we constructed the gene interaction network shared by STP1 and STP2 (Fig. 5). In the previous study, MUP1, FIT2, and YGP1 were only detected from mean change in the double deletion of STP1 and STP $2^{4,5}$. Namely, they cannot be detected in single deletions of STP1 or STP2. However, in this study, those hidden interactions could be detected by the noise-only change, even from the single deletion of STP2. In addition, several new STP2 downstream genes were detected by noise change. We found that some of these were also downstream genes of STP 1 , which supports that they are redundantly regulated by $S T P 1 / 2$ (genes indicated by blue and red arrow in Fig. 5). Here, we discuss about the reason why those novel STP2 downstream genes that have redundant interaction with $S T P 1$, had not been found by $\triangle \mathrm{STP} 1 \triangle \mathrm{STP} 2$ nor $\triangle \mathrm{STP} 2$ in the previous study. If the effect of $S T P 1$ is much larger than that of $S T P 2$ in redundant pathway, the mean expression change of downstream genes estimated in $\triangle \mathrm{STP} 1 \Delta \mathrm{STP} 2$ may not differ from that in $\triangle \mathrm{STP} 1$ due to the subtle effect of STP2. Namely, the detection of asymmetric functional compensation is difficult, even from the double deletion, unless we use noise-only change.

Conventionally, redundantly regulated genes are detected by assessing mean expression change in the double deletion of redundant genes, in order to avoid functional compensation. From this analysis, we propose that redundantly regulated genes can be detected by assessing noise-only change, even in the single deletion of one of the redundant genes. Our new approach will provide a novel framework for revealing complex gene networks. As a limitation of this study, because it is necessary to quantify the variation of expression levels in homogeneous cells, as we showed in this study (Fig. S1 and see Materials and Methods), its application is limited to unicellular organisms at this stage. Furthermore, the quantified noise includes intrinsic noise and extrinsic noise, which later one is not our interests. More accurate interaction analysis should be possible by eliminating extrinsic noise 


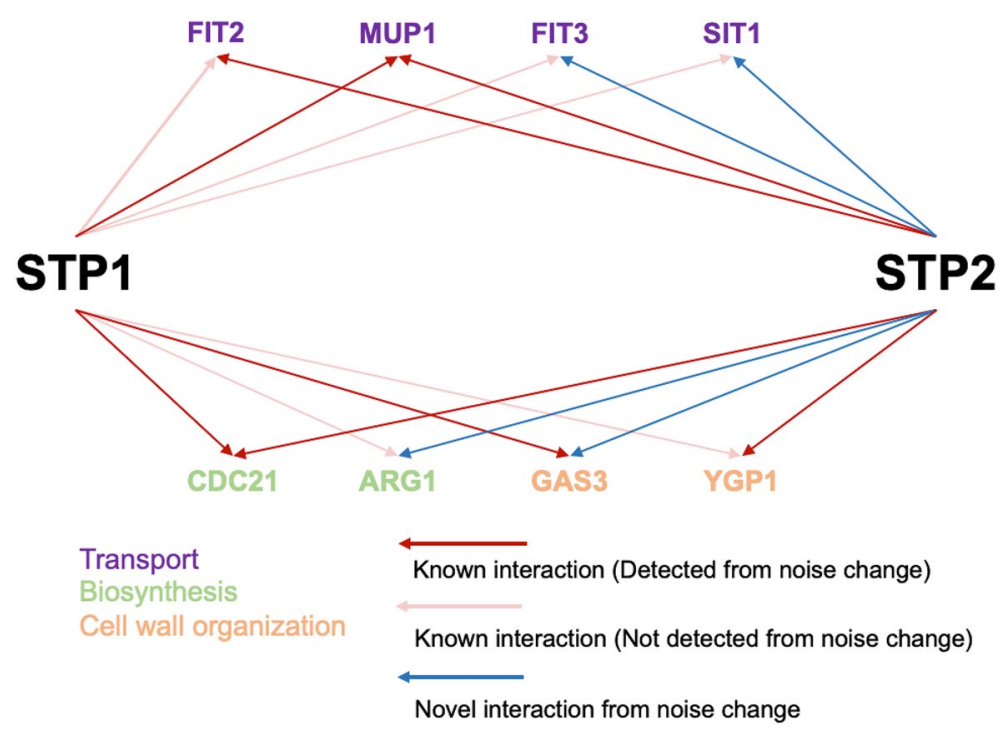

Figure 5. Novel and known interactions shared by STP1 and STP2. Red and blue arrows imply known and novel interactions, respectively. Light red arrows represent known interactions that cannot be detected from noise changes in this study. The color of gene names implies GO terms retrieved from SGD GO Slim mapper.

\begin{tabular}{|c|c|c|}
\hline Name & Interaction & GO term \\
\hline \multicolumn{3}{|l|}{ STP1 } \\
\hline $\mathrm{BIO} 2$ & Novel & Vitamin metabolic process, monocarboxylic acid metabolic process \\
\hline RPL26A & Novel & Cytoplasmic translation, ribosomal large subunit biogenesis \\
\hline ILV5 & Novel & Cellular amino acid metabolic process, mitochondrion organization \\
\hline CLN1 & Novel & Protein phosphorylation, mitotic cell cycle, regulation of protein modification process, regulation of cell cycle \\
\hline WSC2 & Novel & Cell wall organization or biogenesis, response to heat \\
\hline MET6 & Known & Cellular amino acid metabolic process \\
\hline MUP1 & Known & Ion transport, amino acid transport, transmembrane transport \\
\hline TOS4 & Known & Cellular response to DNA damage stimulus \\
\hline GAS3 & Known & Cell wall organization or biogenesis, carbohydrate metabolic process \\
\hline CDC21 & Known & Nucleobase-containing small molecule metabolic process \\
\hline \multicolumn{3}{|l|}{ STP2 } \\
\hline TIM9 & Novel & Mitochondrion organization, protein targeting \\
\hline SIT1 & Novel & Ion transport, transmembrane transport, cellular ion homeostasis \\
\hline NOP16 & Novel & rRNA processing, ribosomal large subunit biogenesis \\
\hline HXT4 & Novel & Ion transport, transmembrane transport, carbohydrate transport \\
\hline GSY2 & Novel & Carbohydrate metabolic process, generation of precursor metabolites and energy \\
\hline RPS17A & Novel & Ribosomal small subunit biogenesis, ribosome assembly, cytoplasmic translation \\
\hline GAS3 & Novel & Cell wall organization or biogenesis, carbohydrate metabolic process \\
\hline ARG1 & Novel & Cellular amino acid metabolic process \\
\hline FIT3 & Novel & Ion transport \\
\hline CIN2 & Novel & Protein folding, cell morphogenesis \\
\hline CLN2 & Novel & $\begin{array}{l}\text { Mitotic cell cycle, protein phosphorylation, response to chemical, regulation of cell cycle, regulation of protein } \\
\text { modification process, conjugation }\end{array}$ \\
\hline MUP1 & Known & Ion transport, transmembrane transport, amino acid transport \\
\hline YGP1 & Known & Cell wall organization or biogenesis \\
\hline CDC21 & Known & Nucleobase-containing small molecule metabolic process \\
\hline FIT2 & Known & Ion transport \\
\hline
\end{tabular}

Table 1. Noise-change genes in $\triangle \mathrm{STP} 1$ and $\triangle \mathrm{STP} 2$. Name: Genes that showed noise change in $\triangle \mathrm{STP} 1$ or $\triangle$ STP2 are listed; Interaction: Noise change genes that had not been reported as STP1 or STP2 downstream in Yeastract are labeled as "Novel"; GO term: GO terms retrieved from SGD GO slim mapper (Yeast GO-Slim process). In the novel STP1 candidates, there were no enriched GO terms (SGD GO term finder process). In the novel STP2 candidates, GO terms were enriched in transport-related genes, in which STP2 downstream genes are involved ( $p<0.01$; SGD GO Term Finder; Process). 
and focusing on intrinsic noise. In this study, we roughly excluded extrinsic noise by clustering scRNA-seq data (Fig. S1, S2). We propose that exact modeling of extrinsic and intrinsic noise using scRNA-seq data will allow for more accurate noise difference estimation. We consider that noise difference tests can be applied as a new strategy for data-driven network estimation that can capture even functional compensation.

\section{Materials and methods}

Expression data. We used single-cell RNA-seq data in yeast, including eight different TF deletion strains $(\triangle \mathrm{STP} 1, \Delta \mathrm{STP} 2, \Delta \mathrm{RTG} 1, \Delta \mathrm{RTG} 3, \Delta \mathrm{GLN} 3, \Delta \mathrm{GAT} 1, \Delta \mathrm{GZF} 3$, and $\triangle \mathrm{DAL} 80)$ and wild type $(\mathrm{WT})$ as a control ${ }^{16}$. $\Delta$ STP1 and $\triangle$ STP2 were used to investigate a redundant gene pair. $\triangle$ RTG1 and $\triangle$ RTG3 were used to investigate a non-redundant gene pair that shares many target genes. $\triangle \mathrm{GAT} 1, \triangle \mathrm{GZF} 3, \triangle \mathrm{DAL} 80$, and $\Delta \mathrm{GLN} 3$ were used to investigate paralogues gene groups, from which redundant functions have not been reported. Each strain had six biological replicates, which were independently constructed and cultured under YPD conditions. Six of the deleted TFs were paralogs (BLASTP search, E-value $<10^{-10}$ ) and two of these were singletons (Table S1). We obtained transcript counts from individual cells, which passed quality control, followed by removal of doublets according to Jackson et al. ${ }^{16}$.

Elimination of cell cycle heterogeneity. In order to precisely quantify the changes in expression noise caused by gene deletion, cell heterogeneity due to cell cycle should be eliminated. Therefore, we divided cells into groups exhibiting similar expression patterns. Two comparative strains were clustered simultaneously. This enabled us to compare two strains within a cluster (Fig. S1). In order to cluster cells by their expression state, first, we normalized cell specific biases using scran normalization methods ${ }^{17}$. Subsequently, we constructed shared nearest neighbor graphs ${ }^{18}$ from normalized counts, and clustered these using Louvain methods ${ }^{19}$. As a result, the strains were grouped into five or six clusters (Fig. S2). Although cells of two strains were randomly distributed in the clusters, expression patterns of $D S E 2^{20}, P I R 1^{21}$, and $H T B^{22}$, known as cell cycle marker genes, were biased by clusters (Fig. S2). Thus, cells were thought to be clustered by cell cycle. Since each cluster represents a specific cell state, two strain comparisons within a cluster was not thought to be affected by heterogeneity due to cell cycle. Since the comparison was executed by cluster, noise (mean) difference results for one gene in a strain equated to the number of clusters. If a gene showed significant change (FDR $<0.01$; Bonferroni correction) in at least one cluster, we considered the gene as a downstream gene candidate of the deleted gene. The results were shown in Fig. S4 and S5. Note that clustering cells based on cell cycle requires a large sample size (number of cells) because the expression pattern in a sampled population is desired to reflect the true populational distribution. Since the sample size of $\triangle$ GLN3 is small, clustering analysis was not executed in that strain. Detected genes in this method are not robust as they are affected by clustering.

Filtration of technical noise. As we described above, each strain includes six biological replicates constructed from different experiments. Note that variance between replicates is smaller than the variance between compared strains (Fig. S6). These replicates were utilized to estimate technical noise under the assumption in which replicate specific variation is technical artifact, and common variation between replicates is biological noise (Fig. 2a; panel 2). Further, Poisson noise is subtracted from the variance as it is interpreted as the RNA sampling noise of the sequencer. As a result, over-dispersion of Poisson noise after accounting for technical noise is considered as biological noise. The example of variance decomposition is shown in the Fig. S6. This filtration was executed using the hierarchical Bayesian model in BASiCS R package $\mathrm{e}^{10,11}$.

Expression level comparison. To test the hypothesis that functional compensation is not detected by mean change but can be detected by noise change, we compared differences in mean expression levels for each gene between wildtype and deletion mutant with differences in expression noise (Fig. 2a). The mean expression level and expression noise were estimated using the hierarchical Bayesian model in BASiCS R package ${ }^{10,11}$. All parameters of MCMC were set to the recommended values in the BASiCS R package manual. Since the expression noise used in the comparison was a residual measure of variability that is not correlated with mean expression level, expression noise change is independent from mean change. The details were reported in Eling et al. ${ }^{10}$ (residual over-dispersion). After comparing the mean with noise respectively, genes showing a significant change with EFDR less than 0.01 were estimated as downstream genes of the deleted gene.

Validation of estimation results. To investigate whether estimated downstream genes were enriched in reported downstream genes, we used the experimentally confirmed interaction database, Yeastract ${ }^{23}$. We retrieved the regulation matrix from Yeastract. Only expression evidence detected under unstressed log-phase growth conditions were included.

Criteria for false negative and false positive targets. In the mean or noise difference tests regarding expression levels, we referred to the false negative target and false positive target. These identifications are based on the Yeastract data which we used as a gold standard. False negative targets are recognized as genes that we could not estimate but have been reported on the Yeastract. On the contrary, genes that we could detect but have not been reported on the Yeastract are possibly novel candidates of downstream genes. However, the newly detected genes that are not related to the known downstream genes or GO terms of known downstream genes are recognized as false positive targets. 


\section{Data availability}

All data analyzed during this study are included in this manuscript. Scripts used in this analysis have been deposited in zenodo (session ID: 5041324).

Received: 30 June 2021; Accepted: 29 October 2021

Published online: 19 November 2021

\section{References}

1. Levine, M. \& Davidson, E. H. Gene regulatory networks for development. Proc. Natl Acad. Sci. USA 102, 4936-4942 (2005).

2. Karlebach, G. \& Shamir, R. Modelling and analysis of gene regulatory networks. Nat. Rev. Mol. Cell Biol. 9, 770-780 (2008).

3. Fiers, M. et al. Mapping gene regulatory networks from single-cell omics data. Brief. Funct. Genomics 17, 246-254 (2018).

4. Eckert-Boulet, N. et al. Transcriptional profiling of extracellular amino acid sensing in Saccharomyces cerevisiae and the role of Stp1p and Stp2p. Yeast 21, 635-648 (2004).

5. Tanaka, N. \& Mukai, Y. Yeast Cyc8p and Tup1p proteins function as coactivators for transcription of Stp1/2p-dependent amino acid transporter genes. Biochem Biophys Res Commun 468, 32-38 (2015).

6. Reimand, J., Vaquerizas, J. M., Todd, A. E., Vilo, J. \& Luscombe, N. M. Comprehensive reanalysis of transcription factor knockout expression data in Saccharomyces cerevisiae reveals many new targets. Nucleic Acids Res. 38, 4768-4777 (2010).

7. Andréasson, C. \& Ljungdahl, P. O. Receptor-mediated endoproteolytic activation of two transcription factors in yeast. Genes Dev. 16, 3158-3172 (2002).

8. Elowitz, M. B., Levine, A. J., Siggia, E. D. \& Swain, P. S. Stochastic gene expression in a single cell. Science 297, 1183-1186 (2002).

9. Blake, W. J., Kaern, M., Cantor, C. R. \& Collins, J. J. Noise in eukaryotic gene expression. Nature 422, 633-637 (2003).

10. Eling, N., Richard, A. C., Richardson, S., Marioni, J. C. \& Vallejos, C. A. Correcting the mean-variance dependency for differential variability testing using single-cell RNA sequencing data. Cell Sys 7, 284-294 (2018).

11. Vallejos, C. A., Marioni, J. C. \& Richardson, S. BASiCS: Bayesian analysis of single-cell sequencing data. PloS Comput Biol 11, e1004333 (2015)

12. Georis, I., Feller, A., Vierendeels, F. \& Dubois, E. The yeast GATA factor Gat1 occupies a central position in nitrogen catabolite repression-sensitive gene activation. Mol Cell Biol. 29, 3803-3815 (2009).

13. Costanzo, M. et al. The genetic landscape of a cell. Science 327, 425-431 (2010).

14. Zheng, J. et al. Epistatic relationships reveal the functional organization of yeast transcription factors. Mol Syst Biol. 6, 420 (2010).

15. Crabeel, M., Seneca, S., Devos, K. \& Glansdorff, N. Arginine repression of the Saccharomyces cerevisiae ARG1 gene. Comparison of the ARG1 and ARG3 control regions. Curr. Genet. 13, 113-124 (1998).

16. Jackson, C. A., Castro, D. M., Saldi, G.-A., Bonneau, R. \& Gresham, D. Gene regulatory network reconstruction using single-cell RNA sequencing of barcoded genotypes in diverse environments. Elife 9, e51254 (2020).

17. Lun, A. T., McCarthy, D. J. \& Marioni, J. C. A step-by-step workflow for low-level analysis of single-cell RNA-seq data with Bioconductor. F1000Res 5, 2122 (2016).

18. Xu, C. \& Su, Z. Identification of cell types from single-cell transcriptomes using a novel clustering method. Bioinformatics 31, 1974-1980 (2015).

19. Blondel, V. D., Guillaume, J.-L., Lambiotte, R. \& Lefebvre, E. Fast unfolding of communities in large networks. J. Stat. Mech. 2008, P10008 (2008).

20. Colman-Lerner, A., Chin, T. E. \& Brent, R. Yeast Cbk1 and Mob2 activate daughter-specific genetic programs to induce asymmetric cell fates. Cell 107, 739-750 (2001).

21. Spellman, P. T. et al. Comprehensive identification of cell cycle-regulated genes of the yeast Saccharomyces cerevisiae by microarray hybridization. Mol Biol Cell 9, 3273-3297 (1998).

22. Eriksson, P. R., Ganguli, D., Nagarajavel, V. \& Clark, D. J. Regulation of histone gene expression in budding yeast. Genetics 191, 7-20 (2012).

23. Monteiro, P. T. et al. YEASTRACT+: A portal for cross-species comparative genomics of transcription regulation in yeasts. Nucleic Acids Res 48, D642-D649 (2020).

\section{Acknowledgements}

We would like to thank Hisao Moriya for valuable comments on our study and all the members of the Makino Laboratory for helpful discussions. This study was supported by KAKENHI (20H03242) from the Japan Society for the Promotion of Science and the Environment Research to T.M.

\section{Author contributions}

T.I. and T.M. conceived and designed the project. T.I. conducted experiments. T.I. and T.M. analyzed the data. T.I. and T.M. wrote the paper.

\section{Competing interests}

The authors declare no competing interests.

\section{Additional information}

Supplementary Information The online version contains supplementary material available at https://doi.org/ 10.1038/s41598-021-01558-y.

Correspondence and requests for materials should be addressed to T.M.

Reprints and permissions information is available at www.nature.com/reprints.

Publisher's note Springer Nature remains neutral with regard to jurisdictional claims in published maps and institutional affiliations. 
(c) (i) Open Access This article is licensed under a Creative Commons Attribution 4.0 International cc) License, which permits use, sharing, adaptation, distribution and reproduction in any medium or format, as long as you give appropriate credit to the original author(s) and the source, provide a link to the Creative Commons licence, and indicate if changes were made. The images or other third party material in this article are included in the article's Creative Commons licence, unless indicated otherwise in a credit line to the material. If material is not included in the article's Creative Commons licence and your intended use is not permitted by statutory regulation or exceeds the permitted use, you will need to obtain permission directly from the copyright holder. To view a copy of this licence, visit http://creativecommons.org/licenses/by/4.0/.

(C) The Author(s) 2021 